\title{
A New Bug on the Block*
}

\author{
Shahid Jameel
}

In December 2019, clusters of pneumonia cases started showing up in the Hubei region of China, especially in its largest city Wuhan. As these piled up, researchers at the Chinese Academy of Sciences' laboratories and the Chinese Centre for Disease Control started looking for the cause. This turned out to be a new virus, whose genetic sequence matched very closely with two coronaviruses isolated from bats in China in 2018.

\section{A Pandemic is Made}

Before public health systems could come to grips with the localized outbreak, people started traveling in large numbers to celebrate the Chinese New Year with their families. This moved the outbreak outside Hubei Region to other parts of China and the world. The rest is history. As on 07 April 2020, there are over 1.3 million confirmed cases, and about 75,000 deaths reported from 184 countries. Almost all new cases are now being registered outside China, particularly in the USA and Europe. On 11th March 2020, the World Health Organization declared it to be a pandemic.

The disease is called Coronavirus Infectious Disease 2019 (Covid19). Though initially called the 2019 novel coronavirus (2019$\mathrm{nCoV}$ ), it was renamed SARS coronavirus 2 (SARS-CoV-2) as the virus closely resembled the one that caused Severe Acute Respiratory Syndrome (SARS) in 2003.

\section{The Outbreak in India}

On 30th January, India's Ministry of Health and Family Welfare

*Vol.25, No.4, DOI: https://doi.org/10.1007/s12045-020-0964-4

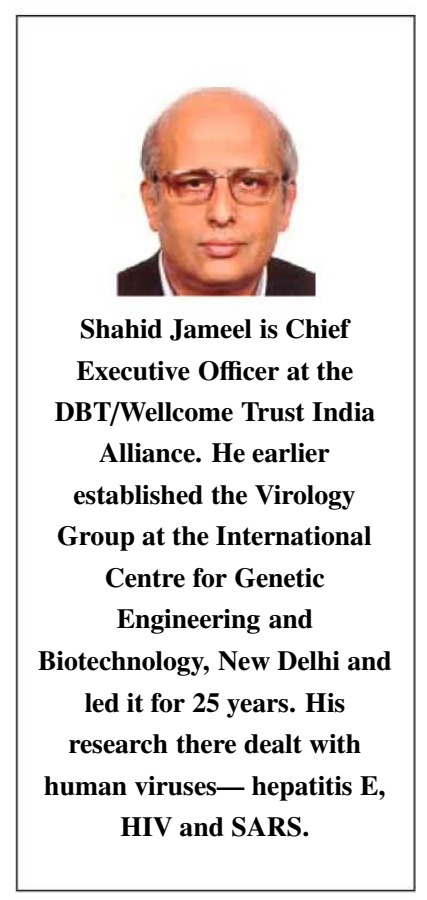

Keywords

Coronavirus, Covid-19, SARS, MERS, RNA polymerase, vaccine. 


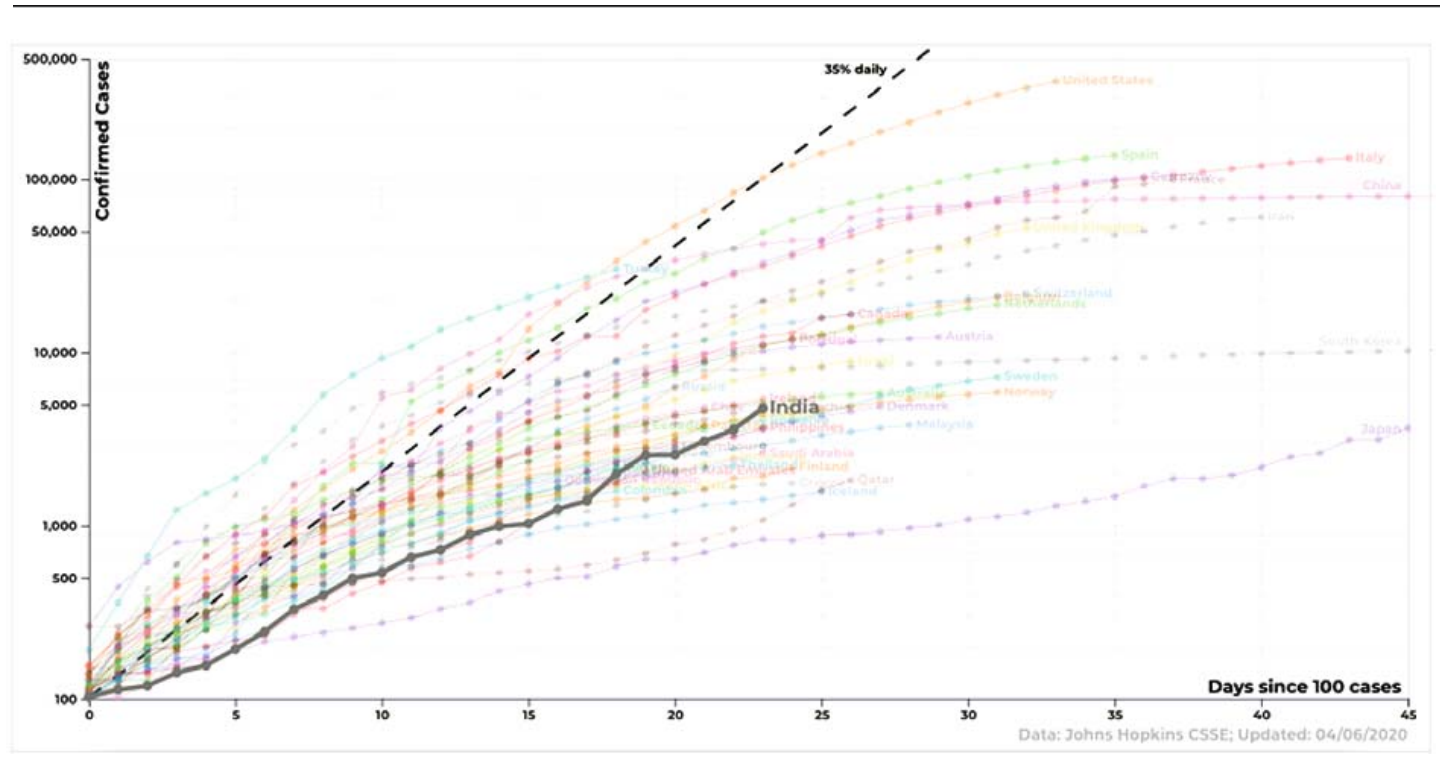

Figure 1. Growth of Covid-19 cases in India, as on 07 April 2020 (Source: http://91-divoc.com/).

There are widely varying estimates for how long the pandemic would continue in India and how many people are likely to get infected. confirmed the infection in a student from Wuhan University, who came home to Kerala. There are now more than 4800 confirmed cases and 136 deaths reported in India. Though the outbreak was initially slow, the cases in India are now doubling every 4 to 5 days (Figure 1). The Government of India has imposed a 3-week nationwide lockdown to reduce transmission. It remains to be seen how well this strategy will work, and if further national, state or region-centric lockdown would be required.

There are widely varying estimates for how long the pandemic would continue in India and how many people are likely to get infected. A model from the Center for Disease Dynamics Economics and Policy at Princeton University, USA, suggests that 30 to 40 crore Indians may get infected by July this year. While most of these cases will be mild, about 1 crore may be severe, and about 20 to 40 lakhs may require hospitalization. Another model by the COV-IND-19 group at the University of Michigan, USA, suggests 13,000 to 21 lakh infections and about 4,000 to 60,000 deaths as best and worst-case scenarios. Most public health experts agree that the period from mid-April to mid-May would be critical for India and that the outbreak would continue till at least 
July with the possibility of a rebound in November/December.

\section{The Biology of Coronaviruses}

Coronaviruses are a group of viruses identified by their crownlike (corona) appearance under a microscope. Members of this family of viruses are known to infect and cause disease in animals and humans. Earlier, six other coronaviruses including the 2003 Severe Acute Respiratory Syndrome (SARS) and the 2012 Middle East Respiratory Syndrome (MERS) viruses, were known to cause disease in humans. Since the SARS outbreak in 2003, scientists have discovered a large number of SARS-related coronaviruses from their natural hosts-bats. Previous studies have shown some of these bat coronaviruses to have the potential to infect humans. The SARS-CoV-2 virus appears to have evolved from one of those.

The SARS-CoV-2 virus particle is about $90 \mathrm{~nm}$ in size, about a million times smaller than the cells that it infects. It carries an RNA genome of about 29,000 nucleotides tightly packed over a scaffold of the viral nucleocapsid protein. This makes the core of the virus, which is then packaged inside a membrane made of lipids and other proteins. The lipid membrane breaks up with soap and water, which is why hand washing protects from infection. The membrane is decorated with three other proteins-the spike protein that sticks out to give a crown-like appearance and the envelope and membrane proteins that sit between the spikes (Figure 2).

The first contact between the virus and its target cell is made by the spike protein binding to a protein called ACE2 found on the surface of human cells, particularly those in the respiratory tract. Once attached, an enzyme TMPRSS2 present on the cell surface cuts the spike protein leading to the fusion of the viral and cellular membranes and delivery of the particle into a subcellular compartment called the endosome. Acidification of these endosomes leads to the fusion of the viral and endosomal membranes and the release of the viral RNA into cells. Once released, the
Since the SARS outbreak in 2003, scientists have discovered a large number of SARS-related coronaviruses from their natural hosts-bats. Previous studies have shown some of these bat coronaviruses to have the potential to infect humans. The SARS-CoV-2 virus appears to have evolved from one of those. 


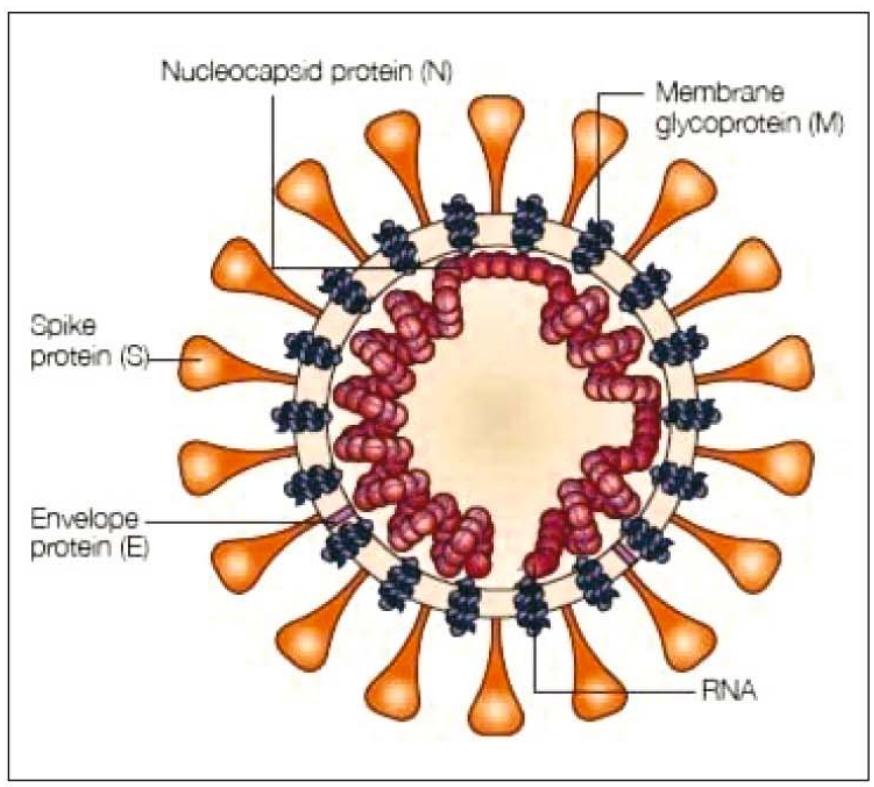

Figure 2. A drawing showing the architecture of a coronavirus.
RNA is translated into proteins - 4 structural proteins, 8 accessory proteins, and a very large replicase or RNA dependent RNA polymerase ( $\mathrm{RdRp}$ ) complex, which is further processed by a viral protease. The RdRp replicates the viral RNA genome to produce more RNAs and through it more proteins. These rounds of amplification produce many new virus particles that are released from infected cells. See Figure 3 for an illustration.

\section{Testing for Covid-19}

The test widely used at this time is called a reverse transcription-polymerase chain reaction (RT-PCR) test. It detects the viral RNA in multiple steps. and blood are also used for testing.
The test widely used at this time is called a reverse transcriptionpolymerase chain reaction (RT-PCR) test. It detects the viral RNA in multiple steps. Usually, nasal aspirates and throat swabs are taken since these are body sites where the virus resides and can be sampled in a non-invasive manner. But lung aspirates, sputum,

Total RNA is extracted from the sample using chemicals that break open the virus, and this RNA is made into a DNA copy (cDNA) using an enzyme called reverse transcriptase. This enzyme itself comes from other viruses called retroviruses. The 


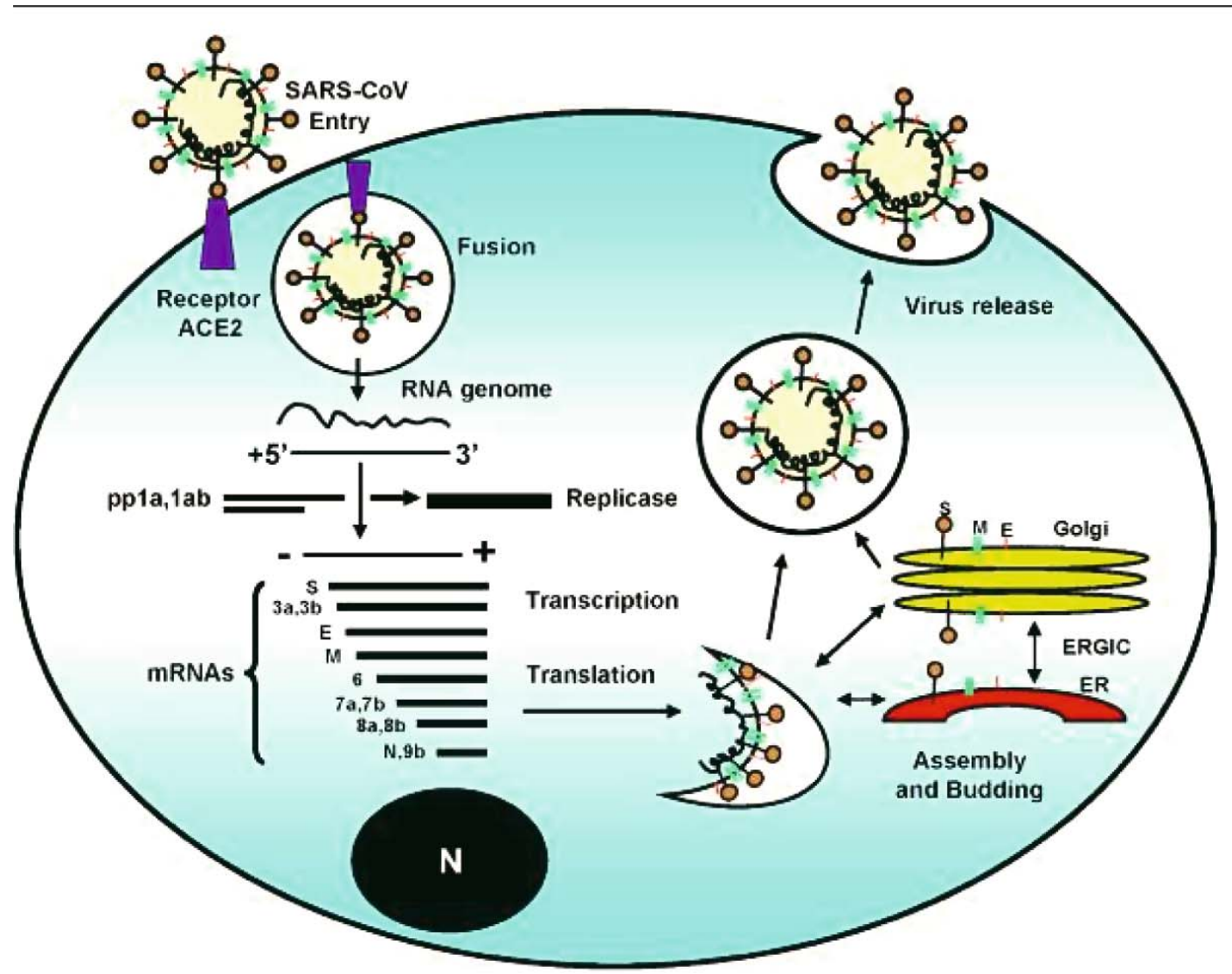

cDNA is then amplified using a process called polymerase chain reaction (PCR), which was discovered in the 1980s. This process uses small bits of single-stranded DNA called primers, which are specific for the SARS-CoV-2 sequence, and a heat-stable enzyme called Taq polymerase to make multiple copies of the cDNA in a very short time. The amplified DNA, which is representative of the viral RNA in the starting sample, is quantified in real-time using a fluorescent dye and compared to negative and positive controls. Starting with total RNA, all the steps from cDNA synthesis to the detection of amplified DNA are carried out in a closed tube inside a real-time PCR machine. It takes a few hours from start to finish, and multiple samples can be analyzed simultaneously.

New testing methods, especially faster ones are being developed.
Figure 3. A view of the life cycle of SARS$\mathrm{CoV}$ (Source: Emerging Microbes and Infections, 1(8): e13, Aug 2012). 
Traditional tests to detect viral proteins (antigen) and antibodies produced in infected people in response to infection are also being developed for Covid-19; these are not yet available for wider use.

\section{Drugs to Treat Covid-19}

There are currently no approved drugs to treat

Covid-19. However, various experimental therapies are being tried in China, Europe, and the USA.

The malaria drug Chloroquine has shown therapeutic value in limited numbers of patients. Chloroquine prevents acidification of endosomes and is known to have broad effects on many viruses that follow the endosome route into cells.
There are currently no approved drugs to treat Covid-19. However, various experimental therapies are being tried in China, Europe, and the USA.

This virus's life cycle can be interrupted at various points. The most obvious target is the replicase since this enzyme is missing from the host cells, which have no interest in replicating their RNA. Nucleotide analogues used as anti-HIV drugs together with protease inhibitors have been tried with some degree of success. A drug that has attracted much attention is Remdesivir, which was originally developed for Ebola but has shown efficacy against other RNA viruses, including the SARS virus. It blocks SARSCoV-2 as well and is now being tested for its efficacy in patients. Another drug being tested is an influenza drug called Favipiravir, which also interferes with the process of making new RNA molecules.

The malaria drug Chloroquine has shown therapeutic value in limited numbers of patients. Chloroquine prevents acidification of endosomes and is known to have broad effects on many viruses that follow the endosome route into cells. The US Federal Drug Administration (FDA), as well as the Government of India, have allowed it to be used in clinical trials as an experimental drug in Covid-19 patients.

Not all drugs work by targeting the virus. Some like interferons work by enhancing the innate immune response and have shown limited efficacy in Covid-19 patients. The virus can also cause damage to the lungs by hyperactivating the immune system's inflammatory response. A molecule called interleukin-6 is involved, and an antibody (Actemra; Tocilizumab) that blocks its receptors on cells has shown therapeutic effect.

About 70 existing drugs and novel chemical entities have been 
identified that may be effective in treating Covid-19. Some of these are already used to treat other diseases, and repurposing them to treat Covid-19, would be faster than developing a drug from scratch.

\section{Covid-19 Vaccines}

A vaccine is a substance that resembles the disease-causing agent (the virus in this case) but does not cause disease. It trains the body's immune system to recognize and kill the virus and creates a memory for the future. There are multiple ways to make viral vaccines. For a killed vaccine, the virus is grown in culture, purified and inactivated with chemicals, for example, the injectable polio vaccine. The virus can be weakened by repeated culture or by genetic means to develop an attenuated strain, such as the one used in the oral polio vaccine. One of the viral surface proteins can also be produced artificially and used to raise immunity, as is the case with the hepatitis $B$ vaccine.

Over 40 different candidate vaccines are already in development for Covid-19. These include an inactivated vaccine being developed in China (Sinovac) using purified SARS-CoV-2, killed with formaldehyde. A genetically engineered strain of SARS-CoV-2 that replicates very poorly is being developed by Codagenix, a US-based company in partnership with the Serum Institute of India (Pune), as a live attenuated vaccine. An experimental vaccine called mRNA-1273 was developed by Moderna, a US biotechnology company and the National Institutes of Health (NIH), USA. A Phase 1 study to evaluate its safety and its ability to raise immune responses in humans was started recently. This vaccine has made history for being the quickest from a virus sequence to start off clinical trials in about two months.

It takes years to develop a vaccine, first in the laboratory, then manufacturing stable and highly pure products to be tested in animals and humans. The Covid-19 vaccine has been fast-tracked into humans with parallel testing in animals. Still, it may take about 18 months for the clinical trials to show whether it is safe
Over 40 different candidate vaccines are already in development for Covid-19. These include an inactivated vaccine being developed in China (Sinovac) using purified SARS-CoV-2, killed with formaldehyde. 
and efficacious. There is also no guarantee at this time that any of the vaccines would work, but scientific knowledge and prior experience tells us that we are on the right track.

\section{What Should We Do?}

This is undoubtedly the biggest public health crisis the world has faced in some time. Since there are no approved therapies and no vaccines, only sensible public health measures will control it. While many other complexities, such as poverty, malnutrition and other diseases, will pose further challenges, we can overcome this pandemic if we remain sensible, disciplined, and sensitive to the needs of those around us.

Infectious disease are also very inclusive. It does not discriminate between religion, caste, economic status, etc. It affects royals, celebrities and commoners alike, and therefore, the fight must involve everyone. It also needs no visas to go across national boundaries, and that is why international cooperation is an effective weapon.

As students of science and as people who believe in evidence and the scientific methods, we must work to educate ourselves and stop the spread of false information. That is the least we can do.

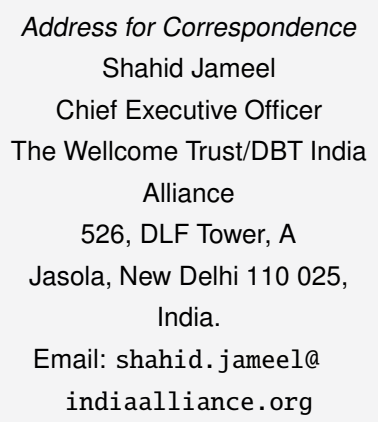

\section{Suggested Reading}

[1] https://www.who.int/health-topics/coronavirus\#tab=tab_1

[2] https://www.mohfw.gov.in/

[3] https://www.youtube.com/watch?v=5DGwOJXSxqg 